ENCYCLOPEDEE Encyclopédie berbère

BERBERE

21 | 1999

21 | Gland - Hadjarien

\title{
Graines sauvages
}

(cueillette en pays touareg)

\section{E. Bernus}

\section{OpenEdition}

\section{Journals}

Édition électronique

URL : http://journals.openedition.org/encyclopedieberbere/1773

DOI : $10.4000 /$ encyclopedieberbere. 1773

ISSN : 2262-7197

\section{Éditeur}

Peeters Publishers

\section{Édition imprimée}

Date de publication : 1 septembre 1999

Pagination : 3199-3208

ISBN : 2-7449-0097-4

ISSN : 1015-7344

\section{Référence électronique}

E. Bernus, «Graines sauvages », Encyclopédie berbère [En ligne], 21 | 1999, document G69, mis en ligne le 01 juin 2011, consulté le 25 septembre 2020. URL : http://journals.openedition.org/ encyclopedieberbere/1773; DOI : https://doi.org/10.4000/encyclopedieberbere.1773

Ce document a été généré automatiquement le 25 septembre 2020.

(c) Tous droits réservés 


\title{
Graines sauvages
}

\author{
(cueillette en pays touareg)
}

\section{E. Bernus}

1 La cueillette peut être définie comme le ramassage des produits spontanés de la nature. Mais il faut distinguer les cueillettes au jour le jour, grappillages d'occasion, qui sont souvent le fait des enfants pour les baies sauvages, des véritables récoltes effectuées collectivement et dont le produit est conservé. Les premières donnent un complément utile en vitamines, les secondes peuvent parfois fournir pendant plusieurs mois la base de l'alimentation d'une famille ou d'un campement.

2 Les récoltes de graines sauvages sont particulièrement développées chez les nomades de la zone sahélienne. Elles peuvent constituer de véritables moissons qui permettent d'éviter l'achat de mil, ou du moins, de le limiter. Ainsi au XIX ${ }^{\mathrm{e}}$ siècle, la confédération des Iwellemmeden Kel Denneg était en lutte constante avec celle des Kel Geres vivant plus au sud, à qui elle contestait la domination de la fertile région de l'Ader-DutchiMajia, riche productrice de mil et de sorgho. Les Kel Geres empêchant les Iwellemmeden de venir recueillir leur part de la récolte de céréales, on rapporte que l' amenokal Musa ag Bodal réunit de nombreuses captives et leur fit récolter des graines sauvages qui furent enfermées dans de grands sacs en cuir et expédiées au chef des Kel Geres, Bodal dit In-Šilkin : par ce geste les Kel Denneg signifiaient qu'ils pouvaient parfaitement se passer de mil et de sorgho, puisque les graines sauvages récoltées dépassaient leurs besoins propres.

3 Cette tradition met en évidence deux faits caractéristiques de cette récolte chez les nomades de la zone sahélienne :

- son importance était telle, au siècle dernier, qu'en bonne année elle permettait, avec le lait, l'autosubsistance ;

- elle était pratiquée par les classes serviles et, parmi elles, surtout par les femmes.

4 Nous verrons plus loin que ces deux faits ont joué un grand rôle dans l'évolution de ces récoltes. 


\section{Les techniques des récoltes}

5 Les techniques varient en fonction des variétés de graines, mais aussi en fonction de l'époque de la récolte.

6 a) Lorsque les nomades cherchent à recueillir la récolte sur pied, c'est-à-dire en fin de saison des pluies, ils peuvent procéder de deux manières :

- à la main : arasab, c'est le fait de presser les épis entre les doigts pour les égrainer : un récipient tenu au-dessous des épis recueille les graines; parfois, une seule secousse ou le choc d'un bâtonnet suffit à les faire tomber.

- au panier : un panier à claire-voie et à fond plein (ekayənkay) balancé à bout de bras recueille les graines qui ne sont pas encore tombées à terre.

7 Ces deux méthodes supposent donc que l'on procède à une récolte précoce, aux mois de septembre et d'octobre. Plus tard, les graines sont à terre et il faut procéder différemment.

8 b) afarad, c'est-à-dire le fait de balayer les graines au sol. C'est la technique la plus couramment utilisée par les nomades : elle permet de procéder à la récolte pendant un temps plus long, et par conséquent d'en faire plusieurs en se déplaçant.

Bellas de l'Ouest nigérien (au Nord d'Ayorou) ramassant les graines d'išiban. L'homme tient le balai à long manche (daji)et la femme le petit balai (azor) (photo E. Bernus).

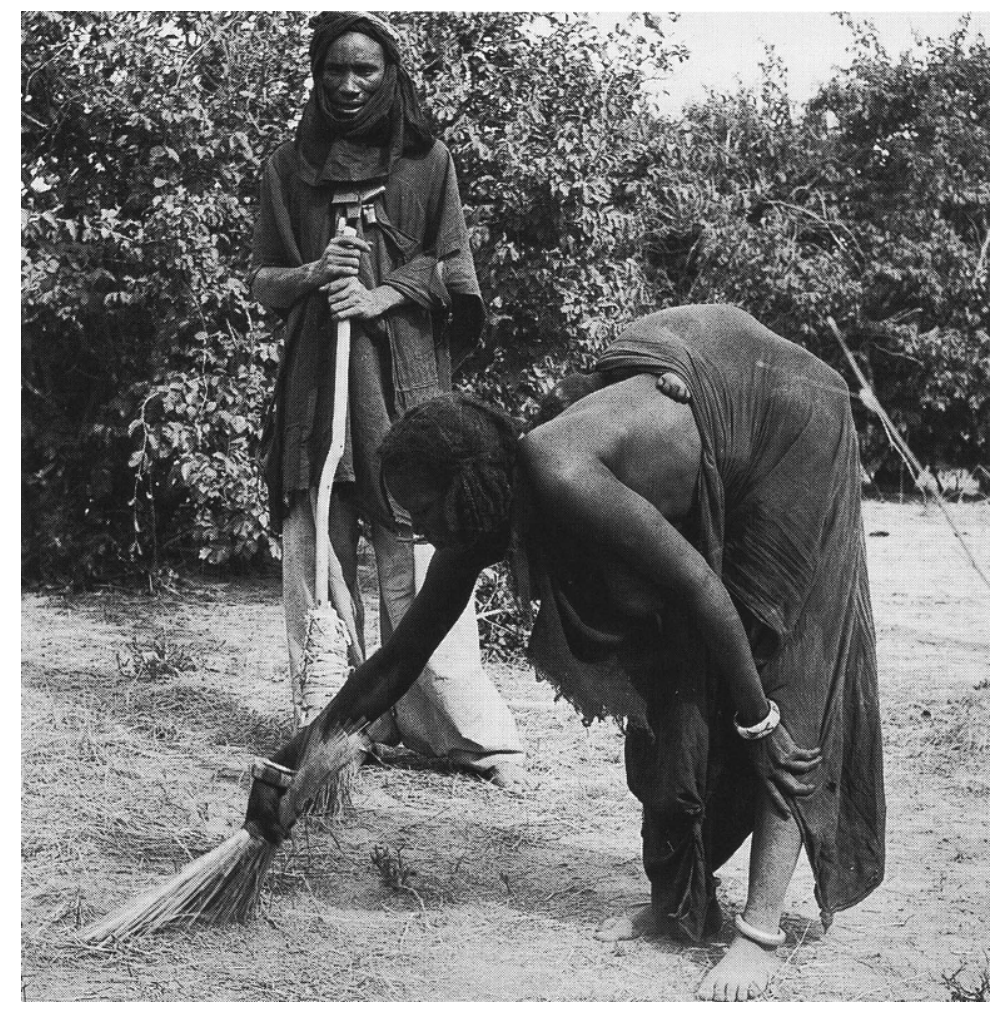

\section{Les graines donnant lieu à de grandes récoltes}

9 - Išiban (plur. sans sing.) sont les graines récoltées en fin de saison des pluies et en saison froide (octobre à décembre), souvent au retour de la transhumance estivale de la "cure salée", nomadisation estivale vers les terres et les sources salées. Ce terme 
désigne en fait de nombreuses graminées; il recouvre les même variétés que le kreb tchadien ou le fonio malien.

- Asyal (Panicum loetum Kunth), la plus appréciée, que l'on trouve surtout dans les basfonds temporairement inondés et akasof qui préfère les terrains plus secs et mieux égouttés.

- Tegabart (Echinochloa colona Link et Eragrostis tremula) aux abords des bas-fonds inondés.

- Tajit (Eragrostis sspp. à petites glumes), également dans les terrains humides.

- Idombisan (Echinochloa colona Link) sur les terrains sableux.

- Uriamum, safari, šiggezal, autres variétés citées par les nomades, la dernière poussant sur les dunes.

Femme Bella vannant les graines ramassées au balai (photo E. Bernus).

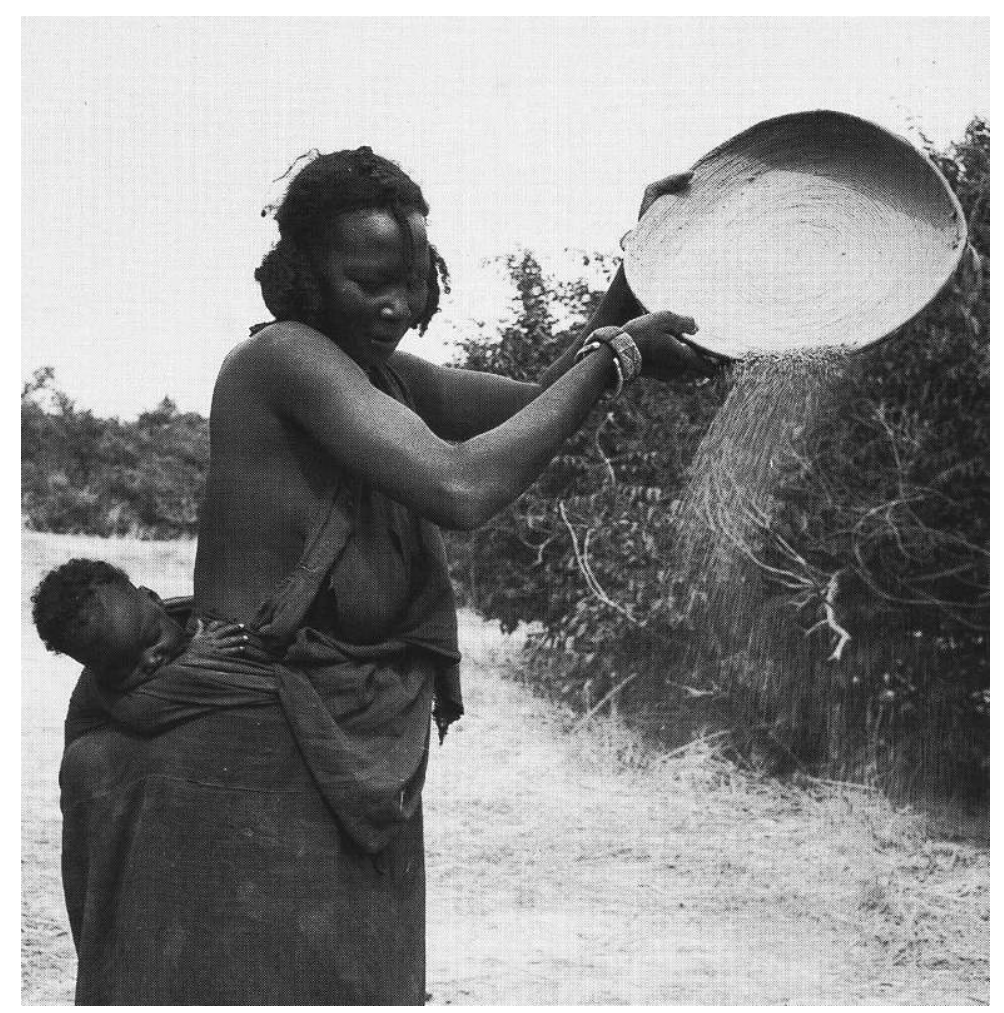

10 La récolte des išiban peut être effectuée au panier assez tôt le matin, pour que la rosée charge les graines d'humidité et les empêche de s'éparpiller au contact du panier. Ce sont surtout les nomades méridionaux, tribus anciennement serves vivant en zone agro-pastorale, qui pratiquent cette technique.

11 La récolte au balai peut succéder à celle au panier : on coupe au couteau la paille, ou on la brûle pour dégager la place. Le balayage est alors effectué par les hommes, au moyen du balais de branchages emmanchés à un long bâton (daji), soit par les femmes avec une touffe de paille, petit balai (azor), semblable à celui qu'elles utilisent pour nettoyer l'intérieur de la tente ou ses abords : elles rassemblent en tas les graines mêlées à de la terre, des cailloux ou des feuilles et destinées aux premiers vannages. Les graines sont amassées pour être ensuite battues avec un lourd pilon (diringi), fait d'un long manche taillé dans un tronc; c'est un outil assez lourd, un pilon élargi à la base, qui vient s'abattre sur les graines disposées sur une aire bien nettoyée. 
12 Au battage ( $u d u z$ ), effectué par les hommes comme par les femmes, succède une série de vannages, travail exclusivement féminin : on cherche à éliminer les corps étrangers par un vannage effectué debout, où les résidus légers s'envolent au fil du vent, et les corps étrangers plus lourds (bâtonnets, cailloux), par un vannage assis. Les femmes procèdent ainsi à une série de vannages, recommencés chacun plusieurs fois, jusqu'à ce que les graines aient été isolées. Alors seulement la récolte peut être engrangée dans de petits greniers de terre, dans des sacs de cuir, dans des silos, ou encore sur des surfaces rocheuses que l'on couvre de paille maintenue par de grosses pierres.

13 Le cram-cram, nom partout retenu, wezzeg ou wejeg (Cenchrus biflorus), est bien connu par ses épines qui s'accrochent aux vêtements avant de pénétrer dans la chair. Les nomades relèvent leurs pantalons au-dessus du genou pour éviter que le tissu ne soit infesté par ses aiguillons. Le cram-cram, qu'ont maudit tous les explorateurs ayant traversé le Sahara, est la plante témoin qui marque la limite entre le Sahara et le Sahel.

Cette récolte s'étale de novembre à mars et peut permettre, dans certains cas, de se passer de toute céréale cultivée. Nous avons visité et filmé au Niger, au nord-ouest de Tchin-Tabaraden, des campements d'affranchis et d'anciens captifs des Arabes Eddès, vivant exclusivement de cram-cram et de lait pendant toute la saison sèche, en se livrant à une récolte collective à laquelle participent tous les habitants.

Le ramassage des graines est effectué par les femmes. Avec un bâton, elles frappent par un mouvement horizontal à ras du sol, la paille pour en faire tomber les graines et les épis. Elles se munissent alors d'un balai à long manche, au bout duquel sont attachées des branches d'épineux. Ce balai (tisidwa c'est-à-dire les branchages), différent de celui qu'utilisent les hommes pour les išiban, sert à rassembler en tas les graines qu'on sépare de la paille. Une natte de lit, disposée sur deux piquets de lit, forme une sorte de brancard sur lequel on entasse les graines épineuses, accrochées encore à des résidus de paille, qui forment une meule. Deux femmes soulèvent le brancard et le posent en équilibre sur la tête d'une troisième qui maintient le tout par les extrémités des piquets, et s'en va vers l'aire de battage, espace nu et dur, dépourvu de végétation.

Le battage commence aussitôt et les femmes viennent se joindre aux hommes lorsque la provision des graines est suffisante pour le travail du jour. Jusqu'à la tombée de la nuit, on entend le lourd battement des pilons frappant le sol; on ramène chaque fois par un mouvement du pied les graines sous le pilon.

On utilise aussi parfois un battoir (billan), épaisse planche de forme arquée, et ayant à son extrémité une poignée faite d'un bâtonnet souple, tendu en arc de cercle par une cordelette qui se fixe dans la planche (cf. Bernus 1967: 35, fig. 2). Cet instrument, réservé aux hommes, sert parfois de complément aux pilons. L'homme tient l'anse souple et fait tournoyer en moulinet le battoir qui s'abat par la tranche sur les graines.

18 Les opérations de vannage qui suivent sont, ici aussi, le lot des femmes.

Wejjeg et išiban donnent lieu à des récoltes quotidiennes qui permettent non seulement d'assurer l'alimentation du campement, mais de constituer des réserves pour plusieurs mois.

Tafa jat (Oryza Barthii A. Chev.), le riz sauvage, est récolté dans de nombreuses mares de la zone sahélienne. C'est un riz annuel, non vivace, récolté en octobre et novembre. Au sud-ouest d'In Gall, au Niger, sur le plateau du Tegama, les Iwellemmeden Kel Denneg procèdent à la récolte lorsque les mares sont encore pleines en arrachant les tiges à la 
main. Plus rarement, on récolte les graines de riz par balayage dans les fonds de mares asséchées.

Si la récolte est importante le riz est battu au bâton sur le sol après séchage. Sinon il est traité directement dans le mortier. Mais les bovins peuls, de plus en plus nombreux dans cette zone, détruisent bien souvent aujourd'hui ces récoltes.

Battage au pilon du cram-cram (Cenchrus biflorus) par des affranchis des Arabes Eddés, au Nord-Est de Tchin-Tabaraden, Niger (photo E. Bernus).

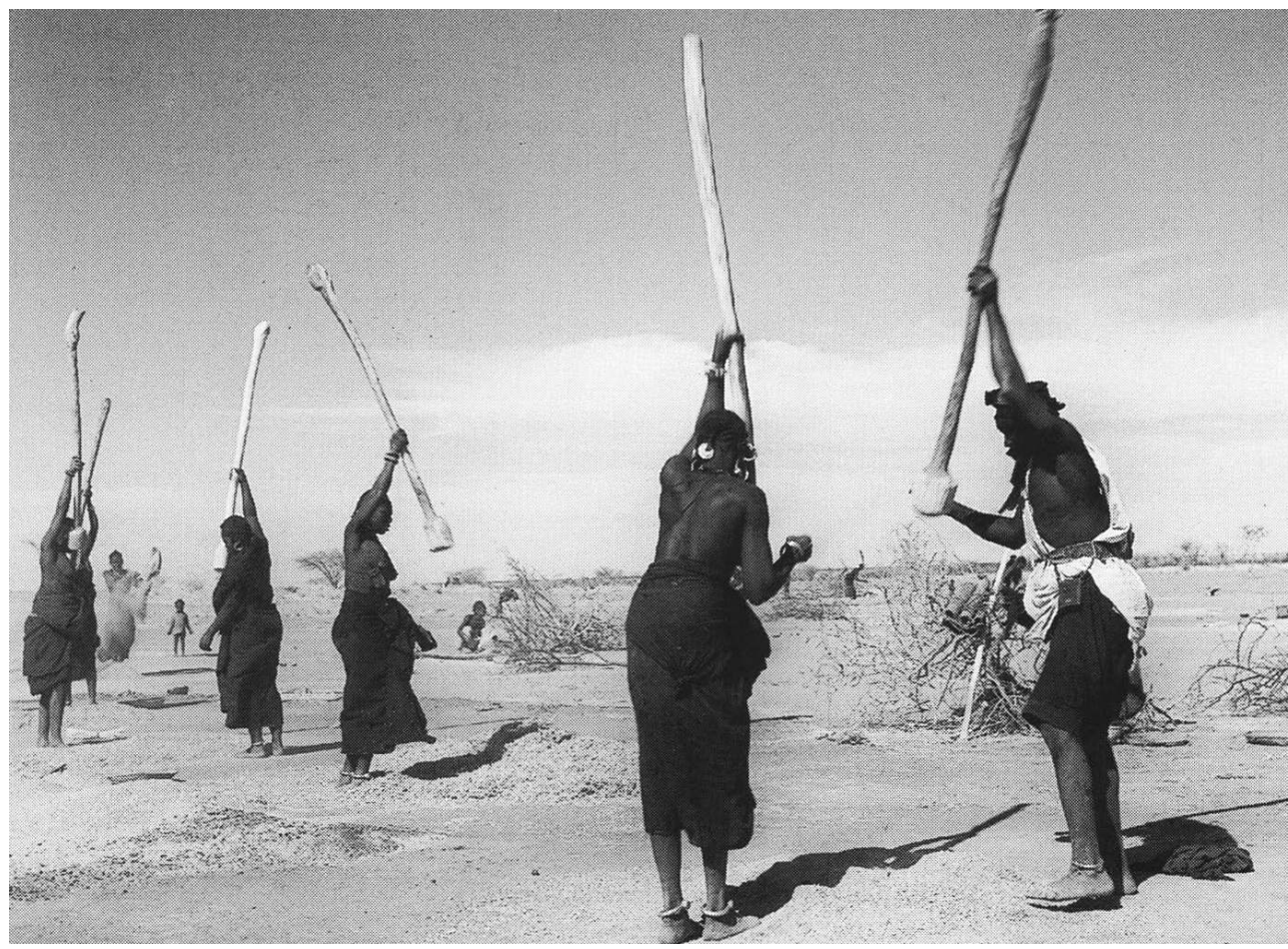

\section{Les graines de complément et de soudure}

piquants moins nombreux et plus courts que ceux du cram-cram, rend la marche très pénible autour des points d'eau où elle est répandue par les troupeaux qui véhiculent ses graines accrochées à leur pelage. Le goût est réputé amer, et on se résout à sa consommation qu'en cas de disette grave; d'où le nom de awetay-n-agarof, l'année de l' agarof pour 1914 et 1931 dans le calendrier des Iwellemmeden Kel Denneg, qui furent des années de sécheresse et de famine.

Ašajor (Sorghum œthiopicum) est un sorgho sauvage récolté à la main. Dans les plaines argileuses de l'Eghazer, à l'ouest de Tegidda-n-Tesemt, aša yor, en bonne année, couvre sans espèces concurrentes d'immenses espaces, au point qu'on dirait des champs de céréales cultivées s'étendant à perte de vue. 

sac en cuir, et un van. Chaque femme s'installe sur une fourmilière et dans le silence déterre les graines qu'elle vanne avant de les rassembler dans le sac. Le silence est indispensable pour une opération qui pourrait provoquer la réaction de génies.

Femme appartenant à la tribu des Igdalen, vannant les graines récoltées dans une fourmilière de la plaine d'Asawas, à l'ouest d'Agadez (photo E. Bernus).

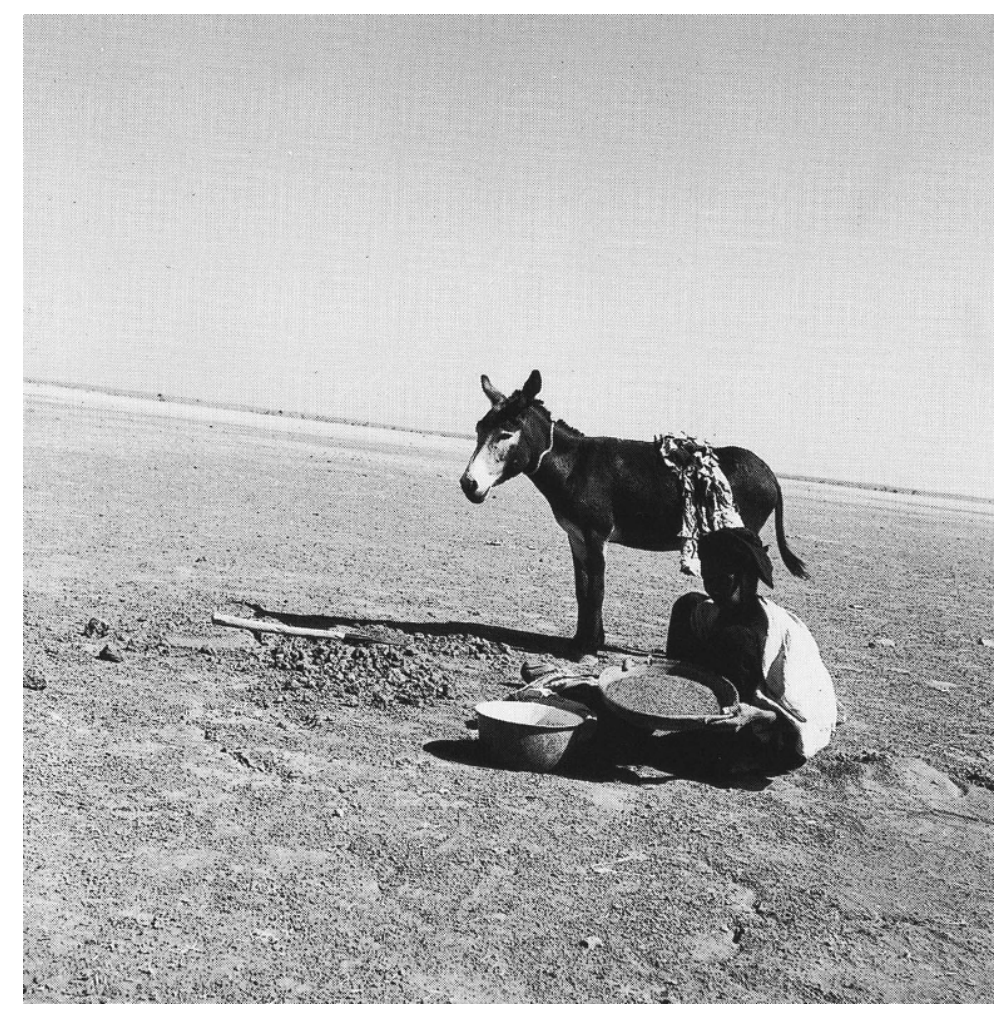

\section{L'évolution}

Les récoltes des graines sauvages, si importantes dans toute la zone saharo-sahélienne, ont tendance à diminuer, pour plusieurs raisons :

Chez les Touaregs et les Arabes, ces récoltes était effectuées par la classe servile. Or on sait que les liens qui existaient entre les serfs et leurs maîtres, entre les tribus anciennement serves, si nombreuses en zone méridionale, et leurs chefs, ont tendance à se relâcher sinon à disparaître. Le nombre de captifs de tente vivant encore en symbiose avec leurs maîtres dans les campements diminue; beaucoup d'entre eux quittent la zone nomade et s'en vont cultiver au sud. La récolte n'est donc plus effectuée comme autrefois par les serviteurs pour leurs maîtres, mais bien souvent aujourd'hui par les ex-captifs à leur propre profit. Ils peuvent encore parfois donner 
une part de leur récolte dans un échange de services ou par habitude. Mais les récoltes les plus importantes que nous avons pu observer chez les nomades, étaient le fait de tribus affranchies ou libérées, très pauvres, qui trouvaient là l'essentiel de leur subsistance.

Toutes les tribus anciennement serves (les Bella de l'Ouest nigérien, du Nord du Burkina Faso, de l'Est du Mali par exemple), qui se sont mises à cultiver extensivement le mil sur une grande échelle, continuent à pratiquer les récoltes de graines sauvages pour leur consommation, réservant à la vente sur les marchés la part la plus importante possible du mil qu'ils produisent. On voit donc que ces cueillettes sont toujours vivantes chez les anciens serfs, vivant pauvrement en zone agro-pastorale et également en zone nomade, qui cherchent à diversifier leur économie. L'éclatement de la société touarègue a fait que désormais les spécialisations qui existaient et dont profitaient les hommes libres jouent surtout à l'intérieur de chaque catégorie sociale.

Graminées sauvages du Sahara.

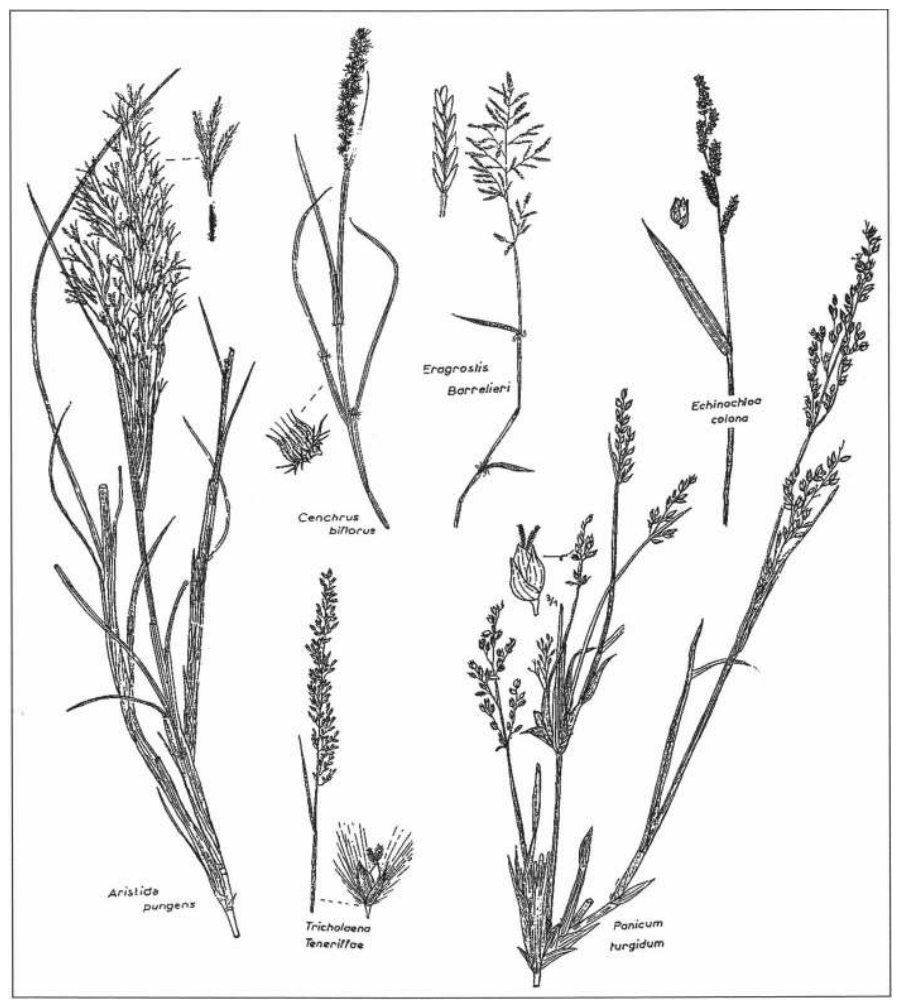

Tricholaena Teneriffae et Echinochloa colona sont absentes du Sahahra central et méridional. Le drinn (Aristida pungens), le millet (Panicum turgidum) et le cram-cram (Cenchrus biflorus) fournissent les récoltes les plus abondantes (dessin P. Ozenda). 


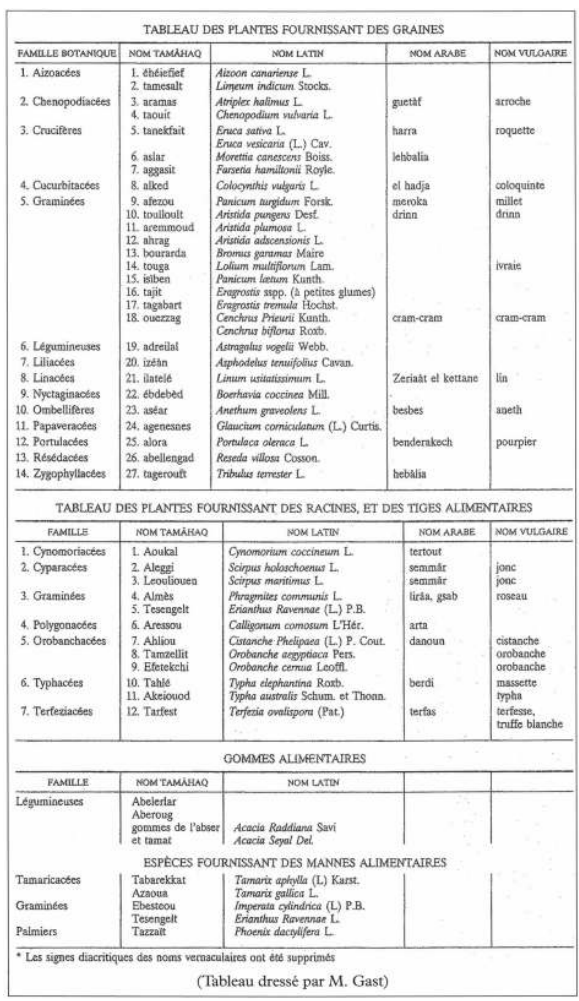

En second lieu, la culture du mil ne peut s'étendre en zone sahélienne et le front pionnier progresse d'année en année vers le nord, au point que le gouvernement nigérien a dû établir une législation pour protéger la zone à vocation pastorale. Le mil est aujourd'hui apporté sur tous les puits par les agriculteurs eux-mêmes, qui reviennent l'échanger contre moutons ou chèvres, ou le vendre contre argent. L'habitude de la consommation du mil et du sorgho est entrée dans tous les campements; et par là, on néglige un travail long et minutieux, qui réclame une main d'œuvre considérable.

Enfin, les Peuls Bororo (qui s'appellent eux-mêmes WoDaaBe), nomades éleveurs de gros troupeaux de bovins, ont envahi depuis une cinquantaine d'années tout le nord de la zone sahélienne jusqu'aux confins du Sahara, région occupée naguère par les seuls Touaregs. Refoulés des zones méridionales par l'évolution démographique, par l'extension des cultures tant vivrières que commerciales (arachide, coton) aboutissant à la réduction ou à la disparition des jachères, ils envahissent les parcours et les prairies septentrionaux: de ce fait, la consommation de fourrage par des troupeaux toujours plus nombreux se fait au détriment du ramassage de graines sauvages destinées à l'alimentation des hommes. 


\section{BIBLIOGRAPHIE}

BERNUS E., 1967, « Cueillette et exploitation des ressources spontanées du Sahel nigérien », in Cahiers ORSTOM, série Sc. Hum., vol. IV, n 1, 1967 : pp. 31-52.

BERNUS E., 1988, « Seasonality, climatic fluctuations, and food supplies (Sahelian nomadic pastoral societies) », in Coping with uncertainty in food supply, I. de Garine \& G A. Harrison éd., Clarendon Press, Oxford : pp. 318-336.

BERNUS E., 1992-93, « Des arbres et des herbes aux marges du Sahara », in Sahara, Preistoria e storia del Sahara, Milan, $\mathrm{n}^{\circ} 5$ : pp. 17-28.

BERNUS E., 1993, Touaregs nigériens. Unité culturelle et diversité régionale d'un peuple pasteur, Paris, L'Harmattan, (cf. Ch. 12, L'utilisation des ressources spontanées, pp. 249-258). I ${ }^{\text {re }}$ édition, ORSTOM, 1981.

BRUNEAU DE MIRÉ Ph., 1956, « Vocabulaire botanique tamajeq » in Bruneau de Miré \& Gillet, Contribution à l'étude de la flore du massif de l'Aïr, J. Agr. Trop. et Bot. Appl. t. III, Paris, pp. 741-760.

CHEVALIER A., 1911, « Le riz sauvage de l'Afrique tropicale », in Journ. Agric. Trop, et Bot. appl, T. II : $1-3$.

CHEVALIER A., 1932, Ressources végétales du Sahara et de ses confins nord et sud, Paris, Muséum d'Histoire Naturelle, Labor. d'Agron. Trop., 256 p.

CRÉAC'H P.-V., 1949, « Les problèmes des mois de disette au Moyen Tchad ». Les aliments végétaux de remplacement, in Conf. Interafric. sur l'alimentation et la nutrition, Dschang, Cameroun : pp. 291-295.

CRÉACH P., 1993, Se nourrir au Sahel. L'alimentation au Tchad 1937-1939, Paris, L'Harmattan, 298 p.

GAST M., 1968, Alimentation des populations de l'Ahaggar. Étude ethnographique, Mémoire du C.R.A.P.E., VIII, Paris, A.M.G, 456 p.

GAST M., 1969, « Persistance proto-historique dans l'alimentation des populations du Sahara central », in Revue de l'Occident musulman et de la Méditerranée, VI, Aix-en-Provence : pp. 89-93.

GAST M., 1972, « Céréales et pseudo-céréales du Sahara central (Ahaggar), I, Usages et valeurs alimentaires de quelques graines du Sahara central ", in Jour. d'Agric. Trop. et de Bot. Appl., t. XIX, $\mathrm{n}^{\circ} 12$, pp. 50-55.

GAST M., « Fourmilière », Encyclopédie berbère, t. XIX, F44, p. 2926-2928.

GAST M., « L'alimentation en période de disette au Sahara central », La Religione della Sete, Centro studi archeologia africana, Milan, 1998, p. 93-107.

GAUDIN-HARDING, F. \& OULD AOUDIA M., 1972, « Céréales et pseudo-céréales du Sahara central (Ahaggar), II, Analyses de quelques graines de la région de l'Ahaggar ", in Journ. d'Agric. Trop. et de Bot. Appl. T. XIX, n 12, pp. 55-58.

NICOLAS F., 1950, Tamesna. Les Ioullemmeden de l'Ouest ou Touareg Kel Dinnik, Paris, Imprimerie Nationale, $270 \mathrm{p}$.

PORTÈRES R., 1958-1959, Les appellations de céréales en Afrique, Journ. Agr. Trop, et de Bot. Appl., t. V, $\mathrm{n}^{\circ} 1-11, \mathrm{t}$. VI, n $1-7$

TUBIANA M.-J., 1969, « La pratique actuelle de la cueillette chez les Zaghawa du Tchad », in Journ. d'Agric. Trop. et de Bot. Appl., T. XVI, nº 2-5 : pp. 55-83. 


\section{FILMOGRAPHIE}

BERNUS E., 1968, Cram-cram, CNRS audiovisuel, $16 \mathrm{~mm}$, couleurs, $12 \mathrm{~min}$.

Produit par le Comité du Film Ethnographique, Musée de l'Homme, Paris, distribué par CNRS audiovisuel, Meudon.

Un campement de nomades du Niger qui vit presque exclusivement de la récolte du cram-cram (Cenchrus biflorus).

INDEX

Mots-clés : Alimentation, Botanique, Sahara 